\title{
Complete response to second-line chemotherapy with sunitinib in a patient with high-risk gastrointestinal stromal tumor: A case report
}

\author{
TSUYOSHI SHIRAKAWA ${ }^{1,2}$, TOMOYA HIRATA ${ }^{1}$, KOSUKE MAEMURA $^{1}$, TOSHIYUKI GOTO $^{1}$, \\ YOSHIYA SHIMAO $^{3}$, KOSUKE MARUTSUKA ${ }^{3}$, YUJI UEDA ${ }^{4}$ and IKUO KIKUCHI ${ }^{1}$ \\ Departments of ${ }^{1}$ Internal Medicine, ${ }^{2}$ Oncology, ${ }^{3}$ Pathology and ${ }^{4}$ Surgery, \\ Miyazaki Prefectural Miyazaki Hospital, Miyazaki 880-8510, Japan
}

Received February 21, 2017; Accepted May 19, 2017

DOI: $10.3892 / \mathrm{mco} .2017 .1268$

\begin{abstract}
Gastrointestinal stromal tumors (GISTs) are a type of sarcoma and the most common mesenchymal tumor of the gastrointestinal tract. Systemic chemotherapy is recommended for unresectable or metastatic GISTs. Imatinib, an oral multi-targeted receptor tyrosine kinase inhibitor, is an effective adjuvant chemotherapy for primary high-risk cases and palliative chemotherapy for unresectable or metastatic cases. For imatinib-resistant cases, second-line chemotherapy with sunitinib is recommended, as it has been demonstrated to result in a significantly longer median progression-free survival time and produce a higher response rate compared with placebo treatment. The current report describes the case of a 54-year-old woman who initially presented with persistent upper abdominal pain and anorexia. Upper gastrointestinal endoscopy and computed tomography revealed a submucosal tumor of the stomach with no apparent metastases. The patient underwent total radical gastrectomy and was diagnosed histologically with GIST with a high-risk of recurrence; therefore, adjuvant chemotherapy with imatinib was administered. However, multiple liver and lymph node metastases were subsequently detected, and the patient therefore received second-line sunitinib therapy. Following two cycles of sunitinib, the liver and lymph node metastases disappeared, and a complete response (CR) was achieved and sustained for at least 9 months. To the best of our knowledge, there have been no such cases of $\mathrm{CR}$ in previous prospective clinical trials examining the effects of sunitinib, and no other case reports of this worldwide. Thus, this study reports an extremely rare
\end{abstract}

Correspondence to: Dr Tsuyoshi Shirakawa, Department of Oncology, Miyazaki Prefectural Miyazaki Hospital, 5-30 Kitatakamatsu-cho, Miyazaki 880-8510, Japan

E-mail: twriver1979@gmail.com

Key words: gastrointestinal stromal tumor, stage IV, sunitinib, second-line chemotherapy, complete response case of a patient with metastatic GIST who achieved CR with sunitinib as second-line chemotherapy.

\section{Introduction}

Gastrointestinal stromal tumors (GISTs) are a type of sarcoma and the most common mesenchymal tumor of the gastrointestinal tract. They arise from the interstitial cells of Cajal, typically occur in older individuals, and are most common in the stomach, followed by the small intestine (1). In Japan, GIST arises most frequently from the stomach, with a prevalence of 1-2 per 100,000 people (2). The majority of GISTs express the protein product of the KIT proto-oncogene, a transmembrane receptor tyrosine kinase (RTK), the activity of which would normally be regulated by the binding of its ligand (stem cell factor). KIT is positively expressed in $~ 95 \%$ of GISTs; therefore, if a tumor is positive for KIT on immunohistochemistry and its cells are morphologically consistent with GIST on hematoxylin and eosin staining, a diagnosis of GIST can be determined (3). CD34 is positively expressed in $70-80 \%$ of GIST cases, and CD34-positive staining can thus indicate a diagnosis of GIST, unless KIT expression is absent (4).

As $>40 \%$ of GISTs are clinically malignant and considered to be metastatic (1,5-7), systemic chemotherapy is indicated in a substantial proportion of GIST cases. Imatinib mesylate is an oral multi-targeted RTK inhibitor that is effective as adjuvant chemotherapy for primary high-risk cases $(8,9)$ and as palliative chemotherapy for unresectable or metastatic cases $(10,11)$.

For imatinib-resistant GIST in unresectable or metastatic cases, second-line chemotherapy is recommended. Sunitinib mesylate is an oral multi-targeted RTK inhibitor whose effects are associated with the blockade of RTK signaling by KIT, platelet-derived growth factor receptors (PDGFRs), vascular endothelial growth factor receptor (VEGFR)-1, VEGFR-2, VEGFR-3, and Fms-like tyrosine kinase-3 receptor (12). In patients with GIST, sunitinib treatment has been shown to lead to a significantly longer median progression-free survival (mPFS) time and a higher response rate (RR) compared with a placebo (12). Continuous daily dosing of sunitinib in GIST patients was also reported to be effective for disease control (13). However, to the best of our knowledge, 
there have been no cases showing complete response (CR) in prospective clinical trials examining the effects of sunitinib for GIST or in case reports worldwide.

The present report describes an extremely rare case involving a patient with metastatic GIST who achieved a CR with sunitinib as second-line chemotherapy. To our knowledge, this is the first case report to describe this outcome.

\section{Case report}

A 54-year-old woman presented with persistent upper abdominal pain and anorexia, and visited a local doctor in March 2015. The patient had a history of surgeries for a myoma of the uterus, and no history of smoking or alcohol consumption. Her family history included no cancers. Abdominal ultrasound revealed a subcutaneous mass of the upper abdomen, and upper gastrointestinal endoscopy showed a submucosal tumor of the posterior wall of the stomach. As GIST of the stomach was suspected, the patient was referred to the Department of Surgery of Miyazaki Prefectural Miyazaki Hospital (Miyazaki, Japan) in April 2015. Abdominal ultrasound demonstrated free space inside the tumor. As the patient's systolic blood pressure was $80 \mathrm{mmHg}$ (normal range, $90-130 \mathrm{mmHg}$ ) and her hemoglobin level was $8.0 \mathrm{~g} / \mathrm{dl}$ (normal range, $12.0-15.0 \mathrm{~g} / \mathrm{dl}$ ), she was diagnosed with hypovolemic shock due to tumor hemorrhage. Since no distant metastases were observed on computed tomography (CT) (Fig. 1), the patient underwent total gastrectomy and distal pancreatectomy with splenectomy for an advanced invasive tumor. Histological examination of the resected specimen revealed rupture and hemorrhage of the tumor, which was $>15 \mathrm{~cm}$ in maximum diameter (Fig. 2A). The tumor consisted of spindle-shaped or polygonal cells with enlarged nuclei (Fig. 2B). Immunohistochemically, the cells were positive for KIT and CD34, and partially positive for smooth muscle actin and p53 (Fig. 2C-E). Staining for S100 and desmin was negative, and the MIB-1 positive rate was $10.07 \%$ (288/2859 cells; Fig. 2F and G). The tumor was resected completely. Since the patient was diagnosed with a high-risk GIST using the modified Fletcher classification (rupture, size $>10 \mathrm{~cm}$, and $>10$ mitoses per 50 high-power fields) (14), adjuvant chemotherapy with imatinib for 3 years was indicated.

The patient was referred to the Department of Oncology of Miyazaki Prefectural Miyazaki Hospital in May 2015. Her general condition was good and organ functions were wellpreserved. Therefore, systemic chemotherapy with imatinib at $400 \mathrm{mg}$ /day was initiated. In June 2015, the dose of imatinib was reduced to $300 \mathrm{mg} / \mathrm{day}$ due to diarrhea of grade 2 according to the Common Terminology Criteria for Adverse Events (CTCAE) v4.0 (15).

However, in November 2015, multiple metastases in the liver and a metastatic lymph node near the celiac artery were detected on CT (Fig. 3A and B). The patient's Eastern Cooperative Oncology Group performance status was 0 , and her vital signs were within normal ranges. Blood testing revealed a decreased red blood cell count of $329 \times 10^{4} / \mu 1$ (normal range, $\left.380-500 \times 10^{4} / \mu \mathrm{l}\right)$ and a decreased hemoglobin level of $10.8 \mathrm{~g} / \mathrm{dl}$ (normal range, 12.0-15.0 g/dl); however, the patient's general condition remained good, and organ functions were preserved. Second-line chemotherapy with sunitinib $(50 \mathrm{mg} / \mathrm{day}$;

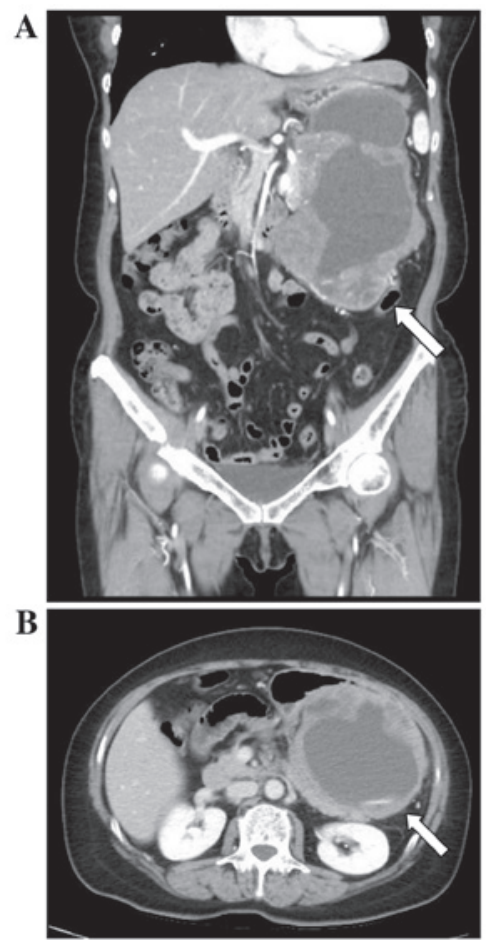

Figure 1. Computed tomography at initial diagnosis revealed (A) a submucosal tumor of the stomach (arrow) with no metastases and (B) tumor hemorrhage (arrow).

days 1-28 per 6-week cycle) was initiated. From the second cycle of chemotherapy, the dose of sunitinib was reduced to $37.5 \mathrm{mg}$ /day due to CTCAE v4.0 grade 1 fatigue. CTCAE v4.0 grade 1 diarrhea and grade 1 proteinuria were also observed, but no other severe adverse events occurred. After two cycles of sunitinib chemotherapy, the liver and lymph node metastases had disappeared, and a partial response, as defined by Response Evaluation Criteria In Solid Tumors version 1.1 (16), was achieved in February 2016. The patient received two additional cycles of sunitinib, and CR was indicated by $\mathrm{CT}$ in May 2016 (Fig. 3C and D). The patient then demanded that chemotherapy be discontinued, despite our recommendation that it be continued. The patient has maintained a CR for 6 months. The most recent follow-up was in March 2017.

\section{Discussion}

In the present case, the patient could be diagnosed with GIST histologically due to the presence of CD34 and KIT immunoreactivity (3). Risk classification is used for GIST cases with no metastases. Historically, the Fletcher classification, which considers tumor size and mitoses, was the first to be used (17). However, it has also been reported that the MIB-1 labeling index and the existence of tumor necrosis are useful as indicators of tumor proliferation (18-20). In addition, since the prognosis of GIST was observed to be dependent on the site of the tumor, the Miettinen classification has been used as a standard for predicting tumor recurrence (21). Furthermore, the modified Fletcher classification, which takes account of tumor size, mitoses, tumor site and tumor rupture, was reported to be more useful for the selection of high-risk recurrent cases compared with the original Fletcher classification (14). The 

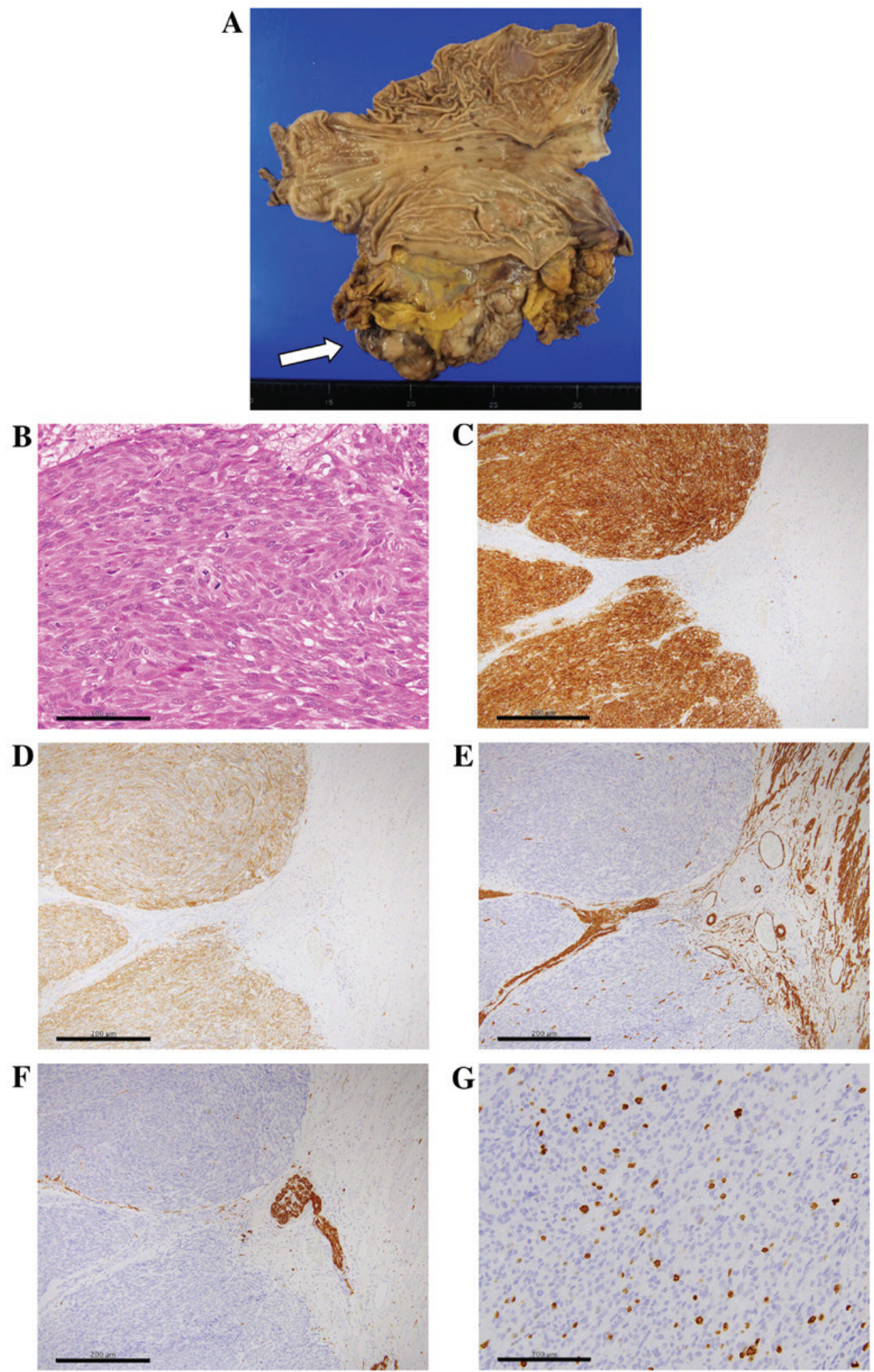

Figure 2. Macroscopic and histological examination of the resected specimen. (A) Macroscopically, the resected tumor was $>15 \mathrm{~cm}$ in maximum diameter. (B) On hematoxylin and eosin staining, the tumor consisted of spindle-shaped or polygonal cells with enlarged nuclei (scale bar, $100 \mu \mathrm{m})$. (C-F) Immunohistochemically, the cells were positive for (C) KIT and (D) CD34, (E) partially positive for smooth muscle actin, and (F) negative for S100 (scale bar, $200 \mu \mathrm{m}$ ). (G) The MIB-1 positive rate was $10.07 \%(288 / 2859$ cells; scale bar, $100 \mu \mathrm{m})$.

present case was diagnosed as high-risk GIST according to the modified Fletcher classification. Although the modified Fletcher classification is a discontinuous indicator for risk assessment, Contour maps are considered to be continuous and useful for the diagnosis of tumor recurrence (14). In the present case, the probability of tumor recurrence was within $80-90 \%$ according to contour maps. Therefore, adjuvant chemotherapy with imatinib was indicated for this case. 

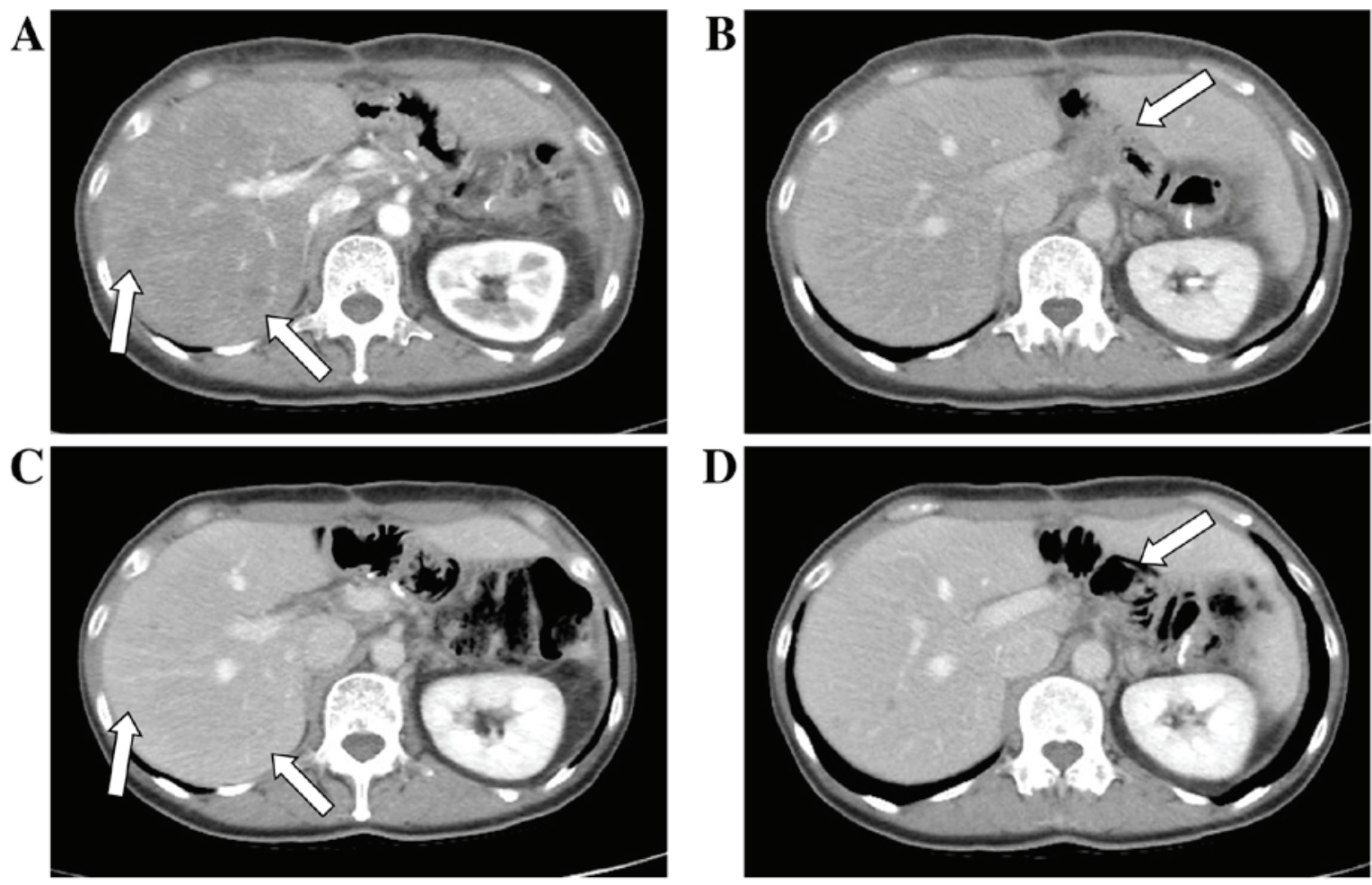

Figure 3. Computed tomography images. In November 2015, following imatinib treatment, (A) multiple metastases (arrows) in the liver and (B) a metastatic lymph node (arrow) near the celiac artery were detected. In May 2016, after 4 cycles of sunitinib, disappearance of (C) the liver metastases (arrows) and (D) the lymph node metastasis (arrow) was observed.

For GIST patients whose tumors are totally resected, imatinib is an effective adjuvant chemotherapy in primary high-risk cases in order to improve the recurrence-free survival time $(8,9)$. For cases of progressive disease in patients receiving $400 \mathrm{mg} /$ day of imatinib therapy, dose escalation to $800 \mathrm{mg} /$ day was reported to be effective in the EORTC62005 trial (22) and the S0033 trial (23). Imatinib dose escalation may have been effective for the current patient; however, $800 \mathrm{mg} /$ day of imatinib is not permitted by the medical insurance system of Japan.

Sunitinib is recommended for imatinib-resistant GIST that is unresectable or metastatic, and significantly longer mPFS times and higher RRs have been observed (12). A previous study conducted a clinical evaluation of continuous daily dosing of sunitinib in patients with advanced GIST following imatinib failure (13). In that study, the disease control rate of sunitinib was $53 \%$, the RR was $13 \%$, the mPFS time was 34 weeks, and the median overall survival (OS) time was 107 weeks. Sahu et al (24) reported no CRs among 15 Indian patients prospectively treated with sunitinib as second-line chemotherapy. Thus, in the prospective clinical trials examining the effects of sunitinib, no cases have achieved CR, to our knowledge.

Retrospective studies reporting CR with second-line chemotherapy involving sunitinib have also been few. Dudeck et al (25) reported no CRs among 51 German patients with GIST. To the best of our knowledge, there have been three cases of CR following sunitinib as second-line chemotherapy for GIST in retrospective studies: 2/199 patients (1.0\%) achieved CR in a Taiwanese study (26), and $1 / 48$ patients (2.1\%) achieved CR in a Chinese study (27). However, to our knowledge, there have been no individual case reports published anywhere worldwide; thus, the present study is the first case report showing CR following second-line sunitinib treatment for GIST.

This case report has several limitations. For example, fluorodeoxyglucose-positron emission tomography (FDG-PET) was not performed. This modality can detect metastatic lesions that are negative on other modalities, and the patient may therefore have had other metastases prior to commencing sunitinib. However, FDG-PET-negative GIST cases can also occur, and FDG-PET is therefore not considered a substitute for CT (28). Furthermore, in the present study, detection of KIT mutation was not performed. Imatinib has a lower reductive effect for GIST cases with a PDGFRA exon 18 D842V mutation (22), and decreased plasma levels of soluble KIT , (compared with the baseline levels) at 20 and 24 weeks after imatinib administration are associated with a prolonged OS time (13). As the four instances of CR were all observed in Asian patients, it is possible that Asian patients may have certain characteristics associated with the effect of sunitinib; however, this requires investigation.

In conclusion, the present case of GIST in which CR was achieved with second-line sunitinib is extremely rare. This information is of importance for the treatment of GIST and also for clinical research.

\section{Acknowledgements}

The authors would like to thank the medical staff involved in this case for their contribution to patient diagnosis and treatment. 


\section{References}

1. Miettinen M and Lasota J: Gastrointestinal stromal tumors-definition, clinical, histological, immunohistochemical, and molecular genetic features and differential diagnosis. Virchows Arch 438: 1-12, 2001.

2. Japanese Clinical Practice Guidelines for Gastrointestinal Stromal Tumors (GIST). 3rd edition. Japan Society of Clinical Oncology, ppl-16, 2004.

3. Hirota S, Isozaki K, Moriyama Y, Hashimoto K, Nishida T, Ishiguro S, Kawano K, Hanada M, Kurata A, Takeda M, et al: Gain-of-function mutations of c-kit in human gastrointestinal stromal tumors. Science 279: 577-580, 1998.

4. Miettinen M, Sobin LH and Sarlomo-Rikala M: Immunohistochemical spectrum of GISTs at different sites and their differential diagnosis with a reference to CD117 (KIT). Mod Pathol 13: 1134-1142, 2000.

5. Pierie JP, Choudry U, Muzikansky A, Yeap BY, Souba WW and Ott MJ: The effect of surgery and grade on outcome of gastrointestinal stromal tumors. Arch Surg 136: 383-389, 2001.

6. DeMatteo RP, Lewis JJ, Leung D, Mudan SS, Woodruff JM and Brennan MF: Two hundred gastrointestinal stromal tumors: Recurrence patterns and prognostic factors for survival. Ann Surg 231: 51-58, 2000.

7. Plaat BE, Hollema H, Molenaar WM, Torn Broers GH, Pijpe J, Mastik MF, Hoekstra HJ, van den Berg E, Scheper RJ and van der Graaf WT: Soft tissue leiomyosarcomas and malignant gastrointestinal stromal tumors: Differences in clinical outcome and expression of multidrug resistance proteins. J Clin Oncol 18 : 3211-3220, 2000

8. Joensuu H, Eriksson M, Sundby Hall K, Hartmann JT, Pink D, Schütte J, Ramadori G, Hohenberger P, Duyster J, Al-Batran SE, et al: One vs three years of adjuvant imatinib for operable gastrointestinal stromal tumor: A randomized trial. JAMA 307: 1265-1272, 2012.

9. Kanda T, Nishida T, Wada N, Kobayashi O, Yamamoto M, Sawaki A, Boku N, Koseki M, Doi T, Toh Y, et al: Adjuvant therapy with imatinib mesylate after resection of primary high-risk gastrointestinal stromal tumors in Japanese patients. Int J Clin Oncol 18 : 38-45, 2013.

10. Demetri GD, von Mehren M, Blanke CD, Van den Abbeele AD, Eisenberg B, Roberts PJ, Heinrich MC, Tuveson DA, Singer S, Janicek M, et al: Efficacy and safety of imatinib mesylate in advanced gastrointestinal stromal tumors. N Engl J Med 347: 472-480, 2002

11. Verweij J, Casali PG,Zalcberg J,LeCesne A, Reichardt P, Blay JY, Issels R, van Oosterom A, Hogendoorn PC, Van Glabbeke M, et al: Progression-free survival in gastrointestinal stromal tumours with high-dose imatinib: Randomised trial. Lancet 364 $1127-1134,2004$.

12. Demetri GD, van Oosterom AT, Garrett CR, Blackstein ME, Shah MH, Verweij J, McArthur G, Judson IR, Heinrich MC, Morgan JA, et al: Efficacy and safety of sunitinib in patients with advanced gastrointestinal stromal tumour after failure of imatinib: A randomised controlled trial. Lancet 368: 1329-1338, 2006.

13. George S, Blay JY, Casali PG, Le Cesne A, Stephenson P, Deprimo SE, Harmon CS, Law CN, Morgan JA, Ray-Coquard I, et al: Clinical evaluation of continuous daily dosing of sunitinib malate in patients with advanced gastrointestinal stromal tumour after imatinib failure. Eur J Cancer 45: 1959-1968, 2009.

14. Joensuu H, Vehtari A, Riihimäki J, Nishida T, Steigen SE, Brabec P, Plank L, Nilsson B, Cirilli C, Braconi C, et al: Risk of recurrence of gastrointestinal stromal tumour after surgery: An analysis of pooled population-based cohorts. Lancet Oncol 13: 265-274, 2012
15. Common Terminology Criteria for Adverse Events (CTCAE) v4.0: Cancer Therapy Evaluation Program, National Cancer Institute.

16. Eisenhauer EA, Therasse P, Bogaerts J, Schwartz LH, Sargent D, Ford R, Dancey J, Arbuck S, Gwyther S, Mooney M, et al: New response evaluation criteria in solid tumours: Revised RECIST guideline (version 1.1). Eur J Cancer 45: 228-247, 2009.

17. Fletcher CD, Berman JJ, Corless C, Gorstein F, Lasota J, Longley BJ, Miettinen M, O'Leary TJ, Remotti H, Rubin BP, et al: Diagnosis of gastrointestinal stromal tumors: A consensus approach. Hum Pathol 33: 459-465, 2002.

18. Wong NA, Young R, Malcomson RD, Nayar AG, Jamieson LA, Save VE, Carey FA, Brewster DH, Han C and Al-Nafussi A: Prognostic indicators for gastrointestinal stromal tumours: A clinicopathological and immunohistochemical study of 108 resected cases of the stomach. Histopathology 43: 118-126, 2003.

19. Fujimoto Y, Nakanishi Y, Yoshimura K and Shimoda T: Clinicopathologic study of primary malignant gastrointestinal stromal tumor of the stomach, with special reference to prognostic factors: Analysis of results in 140 surgically resected patients. Gastric Cancer 6: 39-48, 2003.

20. Hasegawa T, Matsuno Y, Shimoda T and Hirohashi S: Gastrointestinal stromal tumor: Consistent CD117 immunostaining for diagnosis, and prognostic classification based on tumor size and MIB-1 grade. Hum Pathol 33: 669-676, 2002.

21. Miettinen $\mathbf{M}$ and Lasota J: Gastrointestinal stromal tumors: Pathology and prognosis at different sites. Semin Diagn Pathol 23: 70-83, 2006.

22. Zalcberg JR, Verweij J, Casali PG, Le Cesne A, Reichardt P, Blay JY, Schlemmer M, Van Glabbeke M, Brown M and Judson IR; EORTC Soft Tissue and Bone Sarcoma Group, the Italian Sarcoma Group; Australasian Gastrointestinal Trials Group: Outcome of patients with advanced gastro-intestinal stromal tumours crossing over to a daily imatinib dose of $800 \mathrm{mg}$ after progression on $400 \mathrm{mg}$. Eur J Cancer 41: 1751-1757, 2005.

23. Blanke CD, Rankin C, Demetri GD, Ryan CW, von Mehren M, Benjamin RS, Raymond AK, Bramwell VH, Baker LH, Maki RG, et al: Phase III randomized, intergroup trial assessing imatinib mesylate at two dose levels in patients with unresectable or metastatic gastrointestinal stromal tumors expressing the kit receptor tyrosine kinase: S0033. J Clin Oncol 26: 626-632, 2008.

24. Sahu A, Godbole S, Jain P, Ghosh J, Shrikhande S, Ramadwar M, Goyal M, Gulia S, Bajpai J, Kembhavi Y, et al: Sunitinib in patients with imatinib-resistant gastrointestinal stromal tumor: A single center experience study. Indian J Cancer 52: 320-323, 2015.

25. Dudeck O, Zeile M, Reichardt P and Pink D: Comparison of RECIST and Choi criteria for computed tomographic response evaluation in patients with advanced gastrointestinal stromal tumor treated with sunitinib. Ann Oncol 22: 1828-1833, 2011

26. Chen YY, Yeh CN, Cheng CT, Chen TW, Rau KM, Jan YY and Chen MF: Sunitinib for Taiwanese patients with gastrointestinal stromal tumor after imatinib treatment failure or intolerance. World J Gastroenterol 17: 2113-2119, 2011.

27. Liu X, Jiang WZ, Guan GX, Chen ZF, Chi P and Lu HS: Efficacy and safety of sunitinib on patients with imatinib-resistant gastrointestinal stromal tumor. Zhonghua Wei Chang Wai Ke Za Zhi 16: 221-225, 2013 (In Chinese).

28. Demetri GD, von Mehren M, Antonescu CR, DeMatteo RP, Ganjoo KN, Maki RG, Pisters PW, Raut CP, Riedel RF, Schuetze S, et al: NCCN Task Force report: Update on the management of patients with gastrointestinal stromal tumors. J Natl Compr Canc Netw 8 (Suppl 2): S1-S41, quiz S42-S44, 2010. 